\title{
Influence of HIV virus in the hospital stay and the occurrence of postoperative complications classified according to the Clavien-Dindo classification and in comparison with the Charlson Comorbidity Index in patients subjected to urologic and general surgery operations. Our preliminary results
}

\author{
Dimitrios Dimitroulis ${ }^{1}$, Georgios Karaolanis ${ }^{2}$, Ioannis Katafigiotis ${ }^{3}$, Ioannis Anastasiou ${ }^{2}$, \\ Viktoria-Varvara Palla ${ }^{2}$, Athanasios Kontos ${ }^{4}$, Mordechai Duvdevani ${ }^{5}$, Konstantinos Kontzoglou ${ }^{1}$ \\ $12^{\text {nd }}$ Department of Surgery, Laikon General Hospital, Medical School of Athens, Greece; \\ $21^{\text {st }}$ Department of Surgery, Vascular Surgery Unit, Laikon General Hospital, Medical School of Athens, Greece; \\ ${ }^{3} 1^{\text {st }}$ University Urology Clinic, Laiko hospital, Medical School of Athens, Greece; \\ ${ }^{4}$ Department of Pathophysiology, Laikon General Hospital, Greece; \\ ${ }^{5}$ Department of Urology, Hadassah Hebrew University Medical Center, Greece.
}

\begin{abstract}
Summary Objectives: From the first time that human immunodeficiency virus (HIV) was discovered, till today both the quality of life and survival expectancy of HIV-infected patients have markedly improved. As the life expectancy of these patients increases due to the use of highly active anti-retroviral therapy (HAART) also increases the number of HIV-positive patient to be subjected to an operation. Different studies have examined the occurrence of complications in this particular group of patients and their possible susceptibility to infections or other complications that could lead to increased hospital stay, morbidity and mortality with controversial results.

Material and methods: We retrospectively analyzed the data of 25 HIV-patients that were subjected to general surgery and urologic operations and we also examined in comparison with the Charlson score and their comorbidities the occurrence of complications and subsequently the possibility of an increase hospital stay due to their HIV infection. Alongside we classified their complications according to the Clavien-Dindo and compared these complications in relation to their Charlson score and $C D 4$ count.

Results: 10/25 (40\%) of the population had prolonged hospital stay and from this population 6 (6/25) (24\%) patients had less than 200 CD4 constituting the AIDS subpopulation. The decline of the CD4 count showed a tendency for the occurrence of a complication and comorbidities to HIV-positive patients seem to affect more the AIDS subpopulation.

Conclusions: Although this is a small retrospective study, we tried to classify our complications according to the ClavienDindo classification and combine the classification to the age adjusted Charlson score index of comorbidities.
\end{abstract}

KEY WORDS: HIV-positive patients and postoperative complications; HIV-positive patients and surgery; HIV-positive patients and Clavien-Dindo; AIDS and postoperative complications; Hospital stay and Surgery to AIDS patients.

Submitted 19 February 2017; Accepted 23 April 2017

\section{INTRODUCTION}

From the first time that human immunodeficiency virus (HIV) was discovered, till today both the quality of life and survival expectancy of HIV-infected patients have markedly improved (1). As the life expectancy of these patients increases due to the use of highly active antiretroviral therapy (HAART) also increases the number of HIV-positive patient to be subjected to an operation (2). Different studies have examined the occurrence of complications in this particular group of patients and their possible susceptibility to infections or other complications that could lead to increased hospital stay , morbidity and mortality with controversial results (2-5).

We retrospectively analyzed the data of 25 HIV-patients that were subjected to general surgery and urologic operations and we also examined in comparison with the Charlson score and their comorbidities the occurrence of complications and subsequently the possibility of an increase hospital stay due to their HIV infection.

Alongside we classified their complications according to the Clavien-Dindo and compared these complications in relation to their Charlson score and CD4 count.

\section{Materials AND methods - Results}

Patients characteristics (Tables 1, 2)

We retrospectively analyzed from the archives of our Clinic (General surgery and Unit of special infections-Laiko Hospital) the data of 25 HIV-positive patients (mean age 54.32 years) that were subjected to general surgery and urologic operations operations (Tables 1, 2).

For the classification of the comorbidities of our patients we used the age-adjusted Charlson score and for the classification of our complications the Clavien-Dindo 
Table 1.

Patients characteristics.

\begin{tabular}{|lc|}
\hline Number of patients & 25 \\
\hline Mean age & 54.32 years (31-74) \\
\hline Mean Charlson score & $4.04(0-11)$ \\
\hline Mean Clavien & $0.6(0-2)$ \\
\hline Mean CD4 count & $390.72(0-1249)$ \\
\hline AIDS/HIV & $9 / 25(36 \%)$ \\
\hline Patients (HIV + AIDS) with prolonged hospital stay & $10 / 25(40 \%)$ \\
\hline Patients with HIV (no AIDS) and prolonged stay & $4 / 25(16 \%)$ \\
\hline Patients with AIDS and prolonged stay & $6 / 25(24 \%)$ \\
\hline Patients with 2 days additional stay & $9 / 25(36 \%)$ \\
\hline Patients with 1 day additional stay & $1 / 25(4 \%)$ \\
\hline
\end{tabular}

classification. The mean CD4 count was 390.72 and 9/25 (36\%) patients (Table 1) had less than 200 CD4 preoperatively constituting the AIDS subpopulation of the HIV positive patients.

\section{Complications (Table 3)}

Our complications are depicted in Table 3.

Twelve out of 25 (48\%) patients had postoperatively fever requiring prolonged hospital stay $\rightarrow(40 \%)$.

Three of these patients had wound infection as the site of the infection and one of them required an opening of the wound at the bedside and a change in the antibiotic from a cephalosporine of $2^{\text {nd }}$ generation to Piperacillin and Tazobactam while the other two responded to the everyday wound care and to the change of the antibiotic as previously.

In total 10 patients (40\%) (Table 1) had prolonged hospital stay due to fever, including the 3 patients with the wound infection.

Seven patients had fever without a specific infection and from the 10 patients with fever 5 of them needed not only antipyretics but also an upgrade of the antibiotic from cephalosporin of $2^{\text {nd }}$ generation to Piperacillin and Tazobactam to stay without a fever while the other 5 responded well only to antipyretics without the need of an antibiotic change.

\section{Table 2.}

Type of operations.

\begin{tabular}{|c|c|c|c|c|}
\hline Typeof operation & Patients & Protocol hospital stay & Operations with the prolonged stay & Prolonged hospital stay \\
\hline $\begin{array}{l}\text { Biopsy of cervical or Inguinal } \\
\text { or axillary lymph nodes }\end{array}$ & 14 & 0 (daycase) & 4 & $\begin{array}{c}3 \text { patients } 2 \text { days ( } 2 \text { additional days } \\
2 \text { AIDS patients } 1 \text { patient } 3 \text { days ( } 3 \text { additional days) }\end{array}$ \\
\hline $\begin{array}{l}\text { Biopsy of cervical or Inguinal or axillary } \\
\text { lymph nodes + radical orchiectomy }\end{array}$ & 1 & 1 & 1 & 1 patient 3 days ( 2 additional days) AIDS patient \\
\hline $\begin{array}{l}\text { Biopsy of cervical or inguinal or axillary } \\
\text { lymph nodes }+ \text { hydrocele }\end{array}$ & 1 & 0 (daycase) & 1 & 1 patient 2 days ( 2 additional days) \\
\hline Radical orchiectomy & 2 & 1 & 1 & 1 patient 3 days ( 2 additional days) AIDS patient \\
\hline Hydrocele & 2 & 0 (daycase) & 1 & 1 patient 2 days ( 2 additional days) \\
\hline Radical nephrectomy & 2 & 3 & 1 & 1 patient 5 days (2 additional days) AIDS patient \\
\hline Perirectal abscess & 2 & 2 & 1 & 1 patient 4 days ( 2 additional days) AIDS patient \\
\hline Circumcision & 1 & 0 (daycase) & 0 & 0 \\
\hline
\end{tabular}

According to the protocol (Table 2) used in the surgery and urology clinic of our hospital the expected hospital stay for the operations of our population is fluctuating between 0 (day cases) to 3 days.

Two patients were subjected to two operations at the same time, one to cervical lymph node biopsy and radical orchiectomy and the other to cervical lymph node biopsy and hydrocelectomy (Table 2). All the comorbidities were classified according to the Charlson score (mean Charlson score 4.04) and the complications were classified according to the Clavien-Dindo Classification of Surgical Complications (mean Clavien 0.6). The highest Clavien score in this population was 3. In total 10/25 (40\%) of the population had prolonged hospital stay and from this population 6(6/25) (24\%) patients had less than 200 CD4 constituting the AIDS subpopulation. Nine out of 25 (36\%) of the total population had 2 additional days of hospital stay and $1 / 25$ (4\%) had 3 additional days of hospital stay.

The operations in which the prolonged stay occurred are depicted in Table 2. All the AIDS patients with the prolonged stay, 6 out of 25 (24\%) had 2 additional days of prolonged stay according to the protocol of the Hospital for the specific operations.
Table 3.

Complications.

\begin{tabular}{|lc|}
\hline Fever & $10 / 25(40 \%)$ \\
\hline Wound Infection & $3 / 25(12 \%)$ \\
\hline
\end{tabular}

\section{Complications in combination}

with Charlson score and Clavien (Tables 4, 5)

Even though there wasn't an absolute relationship between the Charlson score and the complications, there was a clear tendency of an occurrence of a complication as the Charlson score was higher. Six of the 10 patients (60\%) (Table 4) with complication had more than five points to the Charlson score.

Three of 5 patients with Clavien 2 had more than 5 points to the Charlson score with the highest having eleven. The lack of an absolute relationship between Charslon score and Clavien can be depicted from the fact that there was $3(3 / 25,12 \%)$ patients with Charlson score 6,9 , and 7 respectively without complication and Clavien 0 , and also there were 2 patients with Charlson score 2 and 3 that had a complication with Clavien 2. 
Table 4.

Charlson and Clavien cassification in HIV patients.

\begin{tabular}{|c|c|c|c|c|c|c|c|c|c|c|c|c|}
\hline $\begin{array}{l}\text { Charlson } \\
\text { Clavien }\end{array}$ & 0 & 1 & 2 & 3 & 4 & 5 & 6 & 7 & 8 & 9 & 10 & 11 \\
\hline 0 & $5(20 \%)$ & $2(8 \%)$ & $4(16 \%)$ & & $1(4 \%)$ & & $1(4 \%)$ & $1(4 \%)$ & $1(4 \%)$ & & & \\
\hline 1 & & & $2(8 \%)$ & & & & & & & $1(4 \%)$ & $1(4 \%)$ & $1(4 \%)$ \\
\hline 2 & & & $1(4 \%)$ & $1(4 \%)$ & & & $1(4 \%)$ & & & $1(4 \%)$ & & $1(4 \%)$ \\
\hline
\end{tabular}

\section{Discussion}

Surgical operations to HIV-positive patients constitute a challenge both for the safety of the surgeon and an uneventful postoperative period. In early decades, surgeons were hesitant to perform elective and emergency procedures to this group of patients due to high complication rates $(3,6)$. The development of

CD4 count, Charlson score, complications and Clavien (Tables 5, 6)

The impact of the CD4 count to the complications and Clavien score showed again a clear tendency but not an absolute relationship. The majority of the AIDS patients that is $6 / 9(66.67 \%)$ had a complication. AIDS population with less than $200 \mathrm{CD} 4$ cell/ $\mu$ l automatically had 6 points to the Charlson score meaning that the impact of the CD4 count of the AIDS population to the Charlson score was very important regardless the existence of other comorbidities to these patients. Even though there were 3 AIDS patients (<200 CD4) with no complication and Clavien 0, the decline of the CD4 cells showed a tendency for the occurrence of a complication and also for a higher Clavien (Table 5). Seven out of 25 (28\%) of the patients with a complication had less than 500 CD4 cells, while only 3 patients (12\%) with more than 500 CD4 cells had a complication one with Clavien 2 and two with Clavien 1. Eleven out of 25 (44\%) patients didn't have both comorbidities and complications and only $4 / 25$ (16\%) had both comorbidities and complications (Table 6).

Comorbidities seem to affect more the AIDS population since from the 4 AIDS patients with comorbidities 3 (75\%) had complications, while from the 4 HIV-positive (No AIDS) patients with comorbidities only one (25\%) had complication (Table 6).

\section{Table 5.}

CD4 count and Clavien comparison in HIV patients

\begin{tabular}{|l|c|c|c|}
\hline $\begin{array}{l}\text { CD4 cell/ } \mathbf{\mu l} \\
\text { Clavien }\end{array}$ & $<\mathbf{2 0 0}$ & $\mathbf{2 0 0 - 5 0 0}$ & $>\mathbf{5 0 0}$ \\
\hline 1 & $3(16 \%)$ & $0(0 \%)$ & $2(8 \%)$ \\
\hline 2 & $3(8 \%)$ & $1(4 \%)$ & $1(4 \%)$ \\
\hline 3 & & & \\
\hline
\end{tabular}

\section{Table 6.}

AIDS-HIV patients, comorbidities and complications.

\begin{tabular}{|ll|}
\hline AIDS patients without comorbidities and without complications & $2 / 25(8 \%)$ \\
\hline AIDS patients with comorbidities without complications & $1 / 25(4 \%)$ \\
\hline AIDS patients without comorbidities and with complications & $3 / 25(12 \%)$ \\
\hline AIDS patients with comorbidities and with complications & $3 / 25(12 \%)$ \\
\hline HIV (NO AIDS) patients without comorbidities and without complications & $9 / 25(36 \%)$ \\
\hline HIV (NO AIDS) patients with comorbidities without complications & $3 / 25(12 \%)$ \\
\hline HIV (NO AIDS) patients without comorbidities and with complications & $3 / 25(12 \%)$ \\
\hline HIV (NO AIDS) patients with comorbidities and with complications & $1 / 25(4 \%)$ \\
\hline
\end{tabular}

HAART in the mid-1990s, presented as the cornerstone for the decrease of the morbidity and mortality rates associated with HIV infection in these patients. Based on this scientific evolution, several studies continued to estimate the postoperative complication rates according to the clinical status of the patient and the type of operation performed. Various risk factors have been implicated and examined for the occurrence of complications to the HIV-patients subjected to operations.

The viral load and the history of opportunistic infections may play a significant role in the patient's assessment. Both of these factors depend on the CD4+ lymphocyte counts, the overall immune status, and the survival rate (7). Several studies demonstrated that the CD4+ lymphocyte count is clinically relevant with the risk stratification in HIV patients. Grubert et al. compared 235 patients with HIV undergoing obstetric and gynecological procedures with 235 HIV-negative patients (8). They came to the conclusion that patients with CD4+ counts of $<200$ cells $/ \mu \mathrm{L}$, had a three to four times greater risk of complications after abdominal procedures. Later, Lin et al. came to support these findings after analysis of 48 HIV-seropositive patients, who underwent abdominal aortic surgical treatment (9). Moreover, they found that CD4+ values $<200$ cells/ $\mu \mathrm{L}$ resulted in an increase in the overall operative morbidity and mortality. We also found a clear tendency of an occurrence of complications as the number of CD4 cells was decreasing (Tables 5, 6). The majority of the AIDS population 6/9 $(66,67 \%)$ had a complication (Table 5).

However, the role of CD4+ count as a significant prognostic factor remains debatable, because not all studies have reproduced these findings. Harrison et al. have reported differences in the incidence of postoperative wound infections based on CD4+ lymphocyte counts (10). Moreover, the CD4+ cell count did not affect the incidence of infection $(r=0.16)$. Even though we had 3 AIDS patients $(<200$ CD4cells/ $\mu \mathrm{L}$ ) with no complication (Table 5) in our study the decrease of the CD4 cells affected both the Charlson score and the occurrence of a complication and as a result the Clavien score (Tables 4-6).

Earlier, Paiement et al. in a retrospective study came to the conclusion that HIV-seropositive patients had a postoperative infection rate of $16.7 \%$ when in the seronegative group the postoperative infection rate was $5.4 \%$ (11). When the 
HIV-patients suffered from open trauma the estimated infection rate increased at $55.6 \%$.

Therefore they came to a conclusion that is important to properly identify and optimize the status of CD4+ deficient patients before any elective surgery. In our study the majority of the AIDS patients 6/9 (66,67\%) (Table 6) had a complication and even though 3 AIDS patients with $<200$ CD4 had no complication and clavien 0, the decline of the CD4 cells showed a tendency for the occurrence of a complication and also for a higher clavien (Table 5).

Another significant point that many studies underline is the correlation between postoperative wound infections and the length of hospital stay. Ferrero et al. in a retrospective study evaluated the complications associated with caesarean section in HIV-infected women (12). Because of a higher incidence of major (pneumonia, anaemia) and minor (wound infections) postoperative complications, the median duration of hospital stay (median value 7 days) was significantly higher in the HIV-infected patients compared with the HIV negative. Later, Drapeau et al. reported that HIV-infected patients who underwent different surgical procedures, had the tendency to develop more surgical site infections (SSI) compared to the general population (13). This complication was the cause for longer hospital stay in this patient group. Morrison et al. supported the previous study coming to the conclusion that HIV-infected patients are more likely to develop certain infectious complications and require a longer hospital stay (median value 7.6 days) (14). In our study $10 / 25$ (40\%) of the population had prolong hospital stay and from this population $6(6 / 25)$ (24\%)patients had less than 200 CD4 constituting the AIDS subpopulation (Table 1). 10/25 (40\%) of our patients had postoperatively fever requiring prolong hospital stay (Tables 1, 2). 3 of these patients had wound infection as the site of the infection and 1 of them required an opening of the wound at the bedside and a change in the antibiotic from a cephalosporine of $2^{\text {nd }}$ generation to Piperacillin and Tazobactam while the other two responded to the everyday wound care and to the change of the antibiotic as previously (Tables 1-3).

HIV patients are more likely to develop infectious complications after surgery due to their immune function deficiency. Pneumonia, anaemia, urinary tract infection, surgical wound infection and sepsis are the most common (12). Also in our study fever of unknown origin mainly 10/25 (40\%) and wound infection were the complications 3/25 (12\%) which occurred more frequently (Table 3). Surgical site infection is the third most frequently reported nosocomial infection and one of the main factors causing patient mortality after surgery (15). The mortality rate of patients with sepsis is $30 \%$ to $40 \%$ and up to $50 \%$ for those with severe sepsis or septic shock $(1,16)$. In an attempt to reduce the postoperative complications in HIV-patients, a recent study reported specific guidelines for treatment (17).

Patients with preoperative CD4+ counts $<200$ cells $/ \mu \mathrm{L}$, antibiotic and antifungal medication (sulfamethoxazole and Fluconazole) should be administrated preoperatively as a prophylaxis against Pneumocystis carinii pneumonia and fungal infection. Moreover, in those with deep incisional or organ space infections, administration of appropriate antibiotics in combination with the removal the purulent tissue, is needed. Patients with incisional infections do not require long term use of antibiotics but their incisions should be kept clean (17). 5 of our 10 patients with fever needed an upgrade to the antibiotic from cephalosporin of $2^{\text {nd }}$ generation to Piperacillin and Tazobactam while the other 5 remained without a fever with the use only of antipyretics. Finally it is important to highlight that the presence of comorbidities though showed a clear tendency to increase the occurrence of a complication (Tables 5, 6) since 6 of the 10 patients (60\%) (Table 4) with complication had more than five points to the charlson score, this wasn't an absolute relationship since $3(3 / 25,12 \%)$ patients with charlson score 6,9 , and 7 respectively had no complication and Clavien 0 , and also 2 patients with Charlson score 2 and 3 that had a complication with Clavien 2. AIDS population was affected more from the presence of comorbidities and 3 out of 4 AIDS patients (75\%) with comorbidities had complications, while 1 out 4 HIV-positive (No AIDS) patients $(25 \%)$ with comorbidities had a complication (Table 6).

\section{Conclusions}

Although this is a small retrospective study, we tried to classify our complications according to the ClavienDindo classification and combine the classification to the age adjusted Charlson score index of comorbidities. Our HIV-Positive patients stayed 2.08 days more than expected, the decline of the CD4 count showed a tendency for the occurrence of a complication and comorbidities to HIV-positive patients seem to affect more the AIDS subpopulation. As far as we have reviewed the literature this is the first paper submitted concerning HIV virus and postoperative complications.

\section{References}

1. Drapeau CM, Pan A, Bellacosa C, Cassola G, et al. Surgical site infections in HIV-infected patients: results from an Italian prospective multicenter observational study. Infection. 2009; 37:455-60.

2. Abalo A, Patassi A, James YE, et al. Risk factors for surgical wound infection in HIV-positive patients undergoing surgery for orthopaedic trauma. J Orthop Surg. 2010; 18:224-7.

3. Emparan C, Iturburu IM, Ortiz J, Mendez JJ. Infective complications after abdominal surgery in patients infected with human immunodeficiency virus: role of CD4+ lymphocytes in prognosis. World J Surg. 1998; 22:778-782.

4. Davis PA, Corless DJ, Gazzard BG, Wastell C. Increased risk of wound complications and poor healing following laparotomy in HIV-seropositive and AIDS patients. Dig Surg. 1999; 16:60-7.

5. Morandi E, Merlini D, Salvaggio A, et al. Prospective study of healing time after hemorrhoidectomy: influence of HIV infection, acquired immunodeficiency syndrome, and anal wound infection. Dis Colon Rectum. 1999; 42:1140-4.

6. Huang WC, Kwon EO, Scardino PT, Eastham JA. Radical prostatectomy in patients infected with human immunodeficiency virus. BJU Int. 2006; 98:303-307.

7.Mellors JW, Rinaldo CR Jr, Gupta P, et al. Prognosis in HIV-1 
infection predicted by the quantity of virus in plasma. Science. 1996; 272:1167-70.

8. Grubert TA, Reindell D, Kastner R, et al. Rates of postoperative complications among human immunodeficiency virus-infected women who have undergone obstetric and gynecologic surgical procedures. Clin Infect Dis. 2002; 34:822-30.

9. Lin PH, Bush RL, Yao Q, et al. Abdominal aortic surgery in patients with human immunodeficiency virus infection. Am J Surg. 2004; 188:690-7.

10. Harrison WJ, Lewis CP, Lavy CB. Wound healing after implant surgery in HIV-positive patients. J Bone Joint Surg Br. 2002; 84:802-6.

11. Paiement GD, Hymes RA, LaDouceur MS, et al. Postoperative infections in asymptomatic HIVseropositive orthopedic trauma patients. J Trauma. 1994; 37:545-51.

12. Ferrero S1, Bentivoglio G. Post-operative complications after caesarean section in HIV-infected women. Arch Gynecol Obstet. 2003; 268:268-273.

13. Morrison CA, Wyatt MM, Carrick MM. Effects of human immunodeficiency virus status on trauma outcomes: a review of the national trauma database. Surg Infect. 2010; 11:41-7.

14. Owens CD, Stoessel K. Surgical site infections: epidemiology, microbiology and prevention. J Hosp Infect. 2008; 70(Suppl 2):3-10.

15. Shapiro NI, Howell M, Talmor D. A blueprint for a sepsis protocol. Acad Emerg Med. 2005; 12:352-359.

16. Cheng B, Xie G, Yao S, et al. Epidemiology of severe sepsis in critically ill surgical patients in ten university hospitals in China. Crit Care Med. 2007; 35:2538-2546.

17. Liu BC, Zhang L, Su JS, et al. Treatment of postoperative infectious complications in patients with human immunodeficiency virus infection. World J Emerg Med. 2014; 5:103-6.

\section{Correspondence}

Dimitrios Dimitroulis, MD

dimitroulisdimitrios@yahoo.com

Konstantinos Kontzoglou, MD

kckont@med.uoa.gr

$2^{\text {nd }}$ Department of Surgery, Laikon General Hospital,

Medical School of Athens, Athens, Greece

Georgios Karaolanis, MD

drgikaraolanis@gmail.com

Ioannis Anastasiou, MD

Viktoria-Varvara Palla, MD

vickypalla21@gmail.com

$1^{\text {st }}$ Department of Surgery, Vascular Surgery Unit,

Laikon General Hospital, Medical School of Athens, Athens, Greece

Ioannis Katafigiotis MD, MLS, PhD, FEBU (Corresponding Author)

katafigiotis.giannis@gmail.com

$1^{\text {st }}$ University Urology Clinic, Laiko Hospital, Medical School of Athens

Ag. Thoma 17 - 11527 Athens, Greece

Athanasios Kontos, MD

medkontos@gmail.com

Department of Pathophysiology, Laikon General Hospital, Athens, Greece

Mordechai Duvdevani, MD

moti_duv@yahoo.com

Department of Urology, Hadassah Hebrew University Medical Center,

Athens, Greece 